Service social

\title{
Les apports de l'organisation communautaire en CSSS aux infrastructures communautaires de développement des communautés
}

\author{
René Lachapelle, Denis Bourque et Dominic Foisy
}

Volume 56, numéro 1, 2010

URI : https://id.erudit.org/iderudit/039775ar

DOI : https://doi.org/10.7202/039775ar

Aller au sommaire du numéro

Éditeur(s)

École de service social de l'Université Laval

ISSN

1708-1734 (numérique)

Découvrir la revue

Citer cet article

Lachapelle, R., Bourque, D. \& Foisy, D. (2010). Les apports de l'organisation communautaire en CSSS aux infrastructures communautaires de développement des communautés. Service social, 56(1), 1-14.

https://doi.org/10.7202/039775ar

\section{Résumé de l'article}

Les organisateurs communautaires des CLSC (OC) ont contribué au développement d'organismes communautaires qui ont maintenant les moyens de soutenir sur leurs propres bases le développement des communautés. Pour certains, la création des centres de santé et de services sociaux (CSSS) est l'occasion de s'interroger sur la pertinence de l'organisation communautaire de service public. Une recherche exploratoire menée dans trois milieux distincts montre que cette pratique demeure significativement contributive, une contribution attendue du milieu communautaire et généralement soutenue par les CSSS. La participation citoyenne et le développement des territoires posent de nouveaux défis que les OC des CSSS peuvent aider à relever en soutenant la liaison entre les CSSS et les infrastructures communautaires de développement des communautés (ICDC) et en offrant un accompagnement de milieu. 


\title{
Les apports de l'organisation communautaire en CSSS aux infrastructures communautaires de développement des communautés
}

René LACHAPELLE, M.S.S. Doctorant en Service social, Université Laval

Professionnel de recherche Chaire de recherche du Canada en organisation communautaire Université du Québec en Outaouais

Denis Bourque, Ph.D. Professeur

Département de travail social et des sciences sociales Chaire de recherche du Canada en organisation communautaire Université du Québec en Outaouais

Dominic Foisy, M.T.S. Doctorant Sc. politique, Université d'Ottawa Professeur Département de travail social et des sciences sociales

\section{Résumé}

Les organisateurs communautaires des CLSC (OC) ont contribué au développement d'organismes communautaires qui ont maintenant les moyens de soutenir sur leurs propres bases le développement des communautés. Pour certains, la création des centres de santé et de services sociaux (CSSS) est l'occasion de s'interroger sur la pertinence de l'organisation communautaire de service public. Une recherche exploratoire menée dans trois milieux distincts montre que cette pratique demeure significativement contributive, une contribution attendue du milieu communautaire et généralement soutenue par les CSSS. La participation citoyenne et le développement des territoires posent de nouveaux défis que les OC des CSSS peuvent aider à relever en soutenant la liaison entre les CSSS et les infrastructures communautaires de développement des communautés (ICDC) et en offrant un accompagnement de milieu.

\begin{abstract}
CLSC's community organizers (CO) have contributed to community agencies that now have the means to support their own participation to community development. Some people view the establishment of CSSS as an opportunity to question the relevance of community organization in public service. An exploratory research conducted in three distinct fields shows that this practice remains a significant contribution, a contribution expected in the communities and generally supported by CSSS. Citizen participation and local development raise new challenges that CO in CSSS can help to address, supporting the link between CSSS and Community Infrastructure for Community Development (CICD) and providing support to local action.
\end{abstract}




\section{INTRODUCTION}

La Chaire de recherche du Canada en organisation communautaire s'intéresse aux conditions de succès et de renouvellement de cette pratique confrontée à la transformation des politiques et des services publics, à celle des communautés ainsi que des mouvements sociaux. C'est dans ce cadre qu'a été formulé le projet d'une recherche sur les apports de l'organisation communautaire en CSSS aux infrastructures communautaires de soutien au développement des communautés.

\section{LE CONTEXTE}

Depuis les débuts des centres locaux de services communautaires (CLSC) en 1972, les services d'organisation communautaire ont significativement contribué au développement de l'action communautaire dans les collectivités québécoises. Stimulant l'innovation en réponse aux besoins des communautés, soutenant au besoin la création de nouvelles ressources, appuyant les organismes communautaires dans leurs revendications de reconnaissance et de soutien financier, les organisateurs et organisatrices communautaires (OC) en CLSC ont dispensé des services de soutien professionnel aux organismes communautaires autonomes et favorisé le développement de concertations et de partenariats entre les acteurs du développement local. Ces rapports généralement marqués par la coopération établissement - milieu font partie de l'identité professionnelle des OC (Foisy, 2004; Favreau et Hurtubise, 1993). La pratique de l'organisation communautaire repose sur une expertise résultant "d'un long apprentissage », qui s'est avérée «rare et importante pour un établissement » (Comeau et al., 2008, p. 136). Au tournant des années 2000 , alors que le réseau de la santé et des services sociaux, dans la foulée du rapport Rochon (Québec, 1988), intègre l'action sur les déterminants de la santé dans sa politique de santé publique, adopte le virage ambulatoire en pleine période de crise des finances publiques (Jetté, 2008) et amorce, avec la commission Clair (Québec, 2000), une nouvelle centralisation du réseau au nom des exigences de la nouvelle gestion publique (Jetté, 2008), l'expertise des OC est-elle encore pertinente? La responsabilité populationnelle dévolue aux CSSS et l'obligation qui leur est faite de coordonner un réseau local de services (RLS) sur leur territoire (L.R.Q. chapitre S-4.2) amènent les directions à être directement sur le terrain pour traiter avec les organismes communautaires : reste-t-il un rôle spécifique aux OC?

Les organismes communautaires, de leur côté, bénéficient maintenant du partenariat institué au cours de la décennie 1991-2001 : reconnaissance formelle des groupes communautaires dans la Loi sur les services de santé et les services sociaux de 1991, reconnaissance formelle de la contribution du communautaire à l'exercice de la citoyenneté au Québec dans la politique adoptée en 2001 et croissance notable du soutien de l'État en termes de financement de base de l'action communautaire autonome au cours de la décennie. Disposant d'un meilleur financement, forts de l'expertise développée dans leur milieu et généralement fédérés dans des regroupements comme les corporations de développement communautaire (CDC), les groupes communautaires sont capables de s'imposer comme acteurs sociaux et 
d'établir un rapport de force politique ${ }^{1}$. De plus, les partenaires et les soutiens de l'action communautaire se sont élargis bien au-delà des services d'organisation communautaire des CLSC. Les programmes de santé publique, ceux des centres locaux de développement (CLD) et des corporations de développement économique communautaire (CDEC) en économie sociale et en développement rural, les mesures des plans de lutte contre la pauvreté, les politiques de développement social des municipalités, notamment, ont entraîné l'embauche d'intervenants dans les nouveaux « métiers du développement local et régional » (Robitaille, 2007).

Il y a maintenant une nouvelle génération d'organismes communautaires issus des démarches de concertation, qui ne sont ni de simples tables de concertation, ni des regroupements ou des fédérations d'organismes communautaires, que nous désignons comme des infrastructures communautaires de développement des communautés (ICDC), un néologisme reposant sur la métaphore des équipements collectifs destinés au bien-être d'une communauté. Ce sont des ensembles communautaires complexes capables de fournir des bases matérielles, humaines et politiques au développement des communautés et qui ont pour caractéristiques de :

- Reposer sur une démarche de concertation entre partenaires locaux ou régionaux (selon le niveau du territoire de mobilisation);

- Réunir des acteurs sociaux généralement communautaires et institutionnels (CSSS, municipalités, ministères, etc.), et parfois privés, qui se constituent en initiative de la société civile;

- Disposer de ressources autonomes de soutien à la fois aux processus de concertation, d'une part, et à la mobilisation et la participation des citoyens et citoyennes, d'autre part;

- Être orientés sur le développement des communautés, donc

- d'intervenir sur un ou des territoires d'appartenance clairement définis;

- dans une perspective de répondre collectivement à des problématiques collectives (méthode d'organisation communautaire);

- en vue d'une amélioration des conditions de vie et de la participation sociale des populations, en particulier celles qui sont défavorisées.

Au Québec, une série d'initiatives correspondent à cette définition. On peut mentionner, à titre d'exemples, les tables de quartier en région métropolitaine (Brunet et Durocher, 2006) et les démarches de revitalisation urbaine intégrée (Thériault, 2004). Sans être exhaustive, la liste comprend des organismes comme Vivre SaintMichel en santé à Montréal; le consortium COMSEP-ÉCOF dans la Démarche des premiers quartiers de Trois-Rivières; les Partenaires pour la rénovation des anciens

1. Certains, comme Fournier (2009), estiment toutefois qu'une grande partie des organismes communautaires s'autocensurent en raison précisément de ce meilleur financement gouvernemental. 
quartiers (PRAQ) de Salaberry-de-Valleyfield; le Projet de revitalisation du quartier Saint-Marc/Christ-Roi de Shawinigan; Ascot en santé en Estrie ou encore la Table des partenaires du développement social de Lanaudière.

Enfin, les politiques progressistes qui ont favorisé le développement des communautés et un large accès aux services de soutien communautaire ne sont plus à l'ordre du jour. Elles apparaissent aujourd'hui comme des acquis sectoriels inscrits dans un projet gestionnaire plutôt que social, héritage du néolibéralisme qui a dominé le dernier quart de siècle. La société civile québécoise est aujourd'hui confrontée au danger de cette réduction des organisations à leurs contributions à un marché des services. Le défi de l'organisation communautaire dans une telle conjoncture est de contribuer à redéfinir le développement des communautés et amorcer une « reprise des débats sur la gouvernance démocratique » (Favreau, 2008, p. 188), faire éclater le fonctionnement en silos et inscrire les enjeux dans une recherche de l'intérêt général. Les OC des CSSS sont-ils en mesure de relever de tels défis?

Les tenants d'une approche militante de l'action communautaire estiment que les OC sont maintenant dédiés à l'application de programmes publics plutôt qu'au travail d'animation sur le terrain et de mobilisation des personnes directement concernées par les problématiques, tâches qui sont pourtant le propre de l'organisation communautaire (Shragge, 2006 et 2007). La mise en place de réseaux locaux de services (RLS) vise surtout l'intégration et la gestion d'une première ligne de services mieux inscrite dans le milieu plutôt que le développement de réponses innovatrices issues des communautés (Ellyson et Bourque, 2009). Si l'organisation communautaire en CSSS peut compter sur la reconnaissance de la profession dans le réseau de la santé et des services sociaux, la responsabilité populationnelle, la standardisation des pratiques et la hiérarchisation des services inscrivent cette pratique dans un cadre nettement plus complexe où le départage des intérêts des communautés et celui des établissements ne passe plus par les mêmes modes de gestion (Foisy et al., 2009).

Dans ce contexte, est-il encore légitime de parler d'une contribution de l'organisation communautaire en CSSS au développement des communautés? Les OC ont-ils un rôle spécifique à jouer sur le terrain du développement local communautaire? Si oui, comment définir leur apport au développement des communautés?

\section{LA DÉMARCHE DE RECHERCHE}

Au départ de la recherche, nous faisions l'hypothèse que l'organisation communautaire en CSSS demeure significativement contributive au développement des communautés et que cette contribution est attendue du milieu communautaire, mais qu'elle se concrétise dans de nouveaux rapports des OC avec les divers acteurs du milieu, tout en demeurant authentiquement de l'organisation communautaire. Comme le démontrent Comeau et al. (2006, p. 19), les OC ont « accentué leur fonction de médiation dans les communautés », répondant ainsi au besoin « de médiation des divers niveaux de langage et d'intérêts portés par les acteurs en présence » (Jetté, 2006, p. 30). Foisy (2004) parle pour sa part d'un déplacement vers les rôles de « leader, consultant organisationnel et intermédiaire » (p. 63) ou « médiateur entre les organisations » (p. 65). 
Un second enjeu nous semblait aller dans le sens d'une pertinence de l'organisation communautaire : la participation citoyenne. Dans le contexte où le développement des communautés devient une stratégie de la Santé publique, «l'interaction entre les citoyens ainsi que les liens de confiance et de réciprocité créés constituent le déterminant le plus important pour améliorer la santé et le bien-être d'une collectivité » (Québec, 2008, p. 62). La participation de citoyennes et citoyens aux « processus de coopération volontaire, d'entraide et de construction de liens sociaux » (Cadre de référence, 2002, p. 16) qui caractérisent le développement des communautés, n'est possible que si un soutien leur permet d'établir des rapports égalitaires avec les autres acteurs sociaux, notamment les experts des administrations publiques. De ce point de vue, le Programme national de santé publique 2003-2012, Mise à jour 2008 pointe des « compétences» personnelles et professionnelles assez caractéristiques de l'organisation communautaire en CSSS, compétences qui

« rendent particulièrement habiles à conjuguer les ressources de plusieurs secteurs d'activité :

- l'engagement envers la population et les diverses clientèles;

- la reconnaissance de l'expertise des autres secteurs;

- une vision stratégique et l'aptitude à saisir les occasions;

- la flexibilité;

- le goût du défi, la volonté de repousser les limites;

- la créativité et l'ouverture à la transformation des pratiques;

- la persévérance » (Québec, 2008, p. 67).

Nous faisons l'hypothèse que les OC des CSSS sont encore cohérents avec le cadre de référence du Regroupement québécois des intervenantes et intervenants en action communautaire en CSSS (RQIIAC) (Lachapelle, 2003) et la description de tâches établie par le MSSS (Québec, 2004b, p. 83) qui identifient leur contribution en termes de soutien professionnel au processus par lequel une communauté identifie ses besoins et y trouve une réponse collective.

\section{NOTES MÉTHODOLOGIQUES}

Pour vérifier cette hypothèse, nous avons entrepris une recherche exploratoire dans trois (3) collectivités distinctes (en milieux métropolitain, urbain et semi-rural) parmi celles où nous savions que des ICDC sont actives. Ce choix visait à relativiser l'impact de facteurs particuliers, comme les programmes de revitalisation propres à la région de Montréal, sur la mesure de l'impact de diverses initiatives de revitalisation intégrée sur l'organisation communautaire en CSSS. Dans chacune de ces collectivités, nous avons sollicité une entrevue avec la direction de l'ICDC, la direction du CSSS responsable de l'organisation communautaire et au moins un OC ayant un mandat 
auprès d'une ICDC. À cet échantillon de base nous avons accepté d'ajouter d'autres personnes de chacune de ces catégories, que nos interlocuteurs nous ont recommandé de rencontrer pour bien comprendre la réalité de leur milieu.

Nous avons donc réalisé dix-sept (17) entrevues semi-directives au printemps 2008 auprès de six (6) responsables d'organismes correspondant à notre définition d'ICDC, sept (7) OC et quatre (4) cadres de trois (3) CSSS. La méthode des entrevues semidirectives convient bien aux personnes habituées à réfléchir leurs choix professionnels (Daunais, 1986), comme c'est le cas pour chacune de ces trois catégories d'intervenants. Les entrevues réalisées ont fait l'objet de transcriptions que nous avons analysées à l'aide du logiciel N'Vivo 7. Le petit nombre d'entrevues indique que nous n'avons pas la prétention de rendre compte de toute la richesse ni de toute la diversité des expériences en cours. Nous estimons cependant avoir recueilli suffisamment d'informations convergentes pour conforter notre hypothèse et identifier certains des apports de l'organisation communautaire aux infrastructures communautaires de développement des communautés.

\section{L'ANALYSE DES RÉSULTATS}

Les ICDC où nous avons mené l'enquête ont trois caractéristiques en commun : elles interviennent dans des communautés mal développées; elles se reconnaissent dans la définition du Réseau québécois de revitalisation intégrée ${ }^{2}$ et sont reconnues par d'importants bailleurs de fonds non publics pour le niveau de cohésion des acteurs sociaux qu'elles permettent d'atteindre; enfin, elles ont des rapports de coopération bien établis avec le CSSS de leur territoire

La création des ICDC répondait dans ces trois milieux, selon les OC et les dirigeants des ICDC, à un plafonnement de l'action communautaire. Cette démarche a permis de réaliser une négociation entre divers acteurs concernés de façon à faire monter d'un cran l'intensité de l'action, à maintenir une vision globale au moment où les concertations ne fonctionnaient plus adéquatement, et à rendre possible une action politique. Les ICDC agissent comme une instance de médiation et de négociation. Elles établissent sur le terrain de nouveaux rapports entre la municipalité, le réseau de la santé et des services sociaux et le milieu communautaire. Elles demeurent toutefois fragiles puisqu'elles dépendent de financements sur mesure, pas nécessairement précaires quant au volume, mais ad hoc et qui n'ont pas la stabilité de programmes universellement accessibles.

Les entrevues mettent en évidence de profondes convergences entre les dirigeants des ICDC et les OC des CSSS. Les dirigeants des ICDC estiment que les OC ne sont pas plongés dans les contraintes de la reddition de comptes à un tiers bailleur de fonds, ce qui leur permet de garder une distance critique, un recul, un dégagement et

2. Même s'il ne s'est formellement constitué que le 28 octobre 2008, donc après notre enquête, le Réseau québécois de revitalisation intégrée est l'aboutissement d'une démarche de plusieurs mois réunissant une douzaine d'initiatives qui s'identifient aux caractéristiques décrites dans une déclaration commune que l'on peut lire à l'adresse suivante :

[http://www.uqo.ca/crcoc/Sections/Publications.htm], dans le cahier 0902 aux pages 59 et suiv. 
leur donne de la latitude pour soulever des questions et apporter une contribution spécifique à leur démarche.

Sur les trois territoires où nous avons mené la recherche, les OC des CLSC ont été à l'époque impliqués dans la mise en place de ces nouvelles infrastructures communautaires de développement des communautés. Ils demeurent, du point de vue des dirigeants des ICDC, des passeurs entre des organisations municipales, publiques et communautaires dont les cultures diffèrent. Ils exercent une fonction de liaison des ICDC avec les établissements parce qu'ils comprennent comment les choses se passent à l'interne des CSSS aussi bien que sur le terrain communautaire. Même dans le contexte où les directions de CSSS sont directement en lien avec celles des ICDC notamment en vertu du mandat d'établir un projet clinique en partenariat avec le réseau local de services -, ce rôle d'agent de liaison demeure pertinent puisque les pratiques des OC sont centrées sur une prise en compte de l'ensemble du milieu plutôt que sur les intérêts particuliers de l'un ou l'autre programme des partenaires. Ils font preuve d'une expertise concernant les déterminants sociaux de la santé et développent souvent leur analyse en partenariat avec les nouveaux acteurs du développement (SADC, CLD, CJE, etc.). Ils forment de nouveaux leaders communautaires et, surtout, ils se distinguent des autres intervenants en développement par leur préoccupation pour la participation citoyenne.

La majorité des OC rencontrés partagent ce point de vue, tout en exprimant une préoccupation quant à la contrainte qu'entraîne la modification de leurs rapports avec la direction de leur CSSS. Alors qu'auparavant la direction d'un CLSC s'attendait à ce que ses OC soient avec les groupes, y compris quand ceux-ci ont des revendications à faire valoir, une telle situation est devenue plus critique depuis l'intégration des CLSC dans les CSSS. En fait, les rapports quotidiens ont été profondément modifiés et ne favorisent plus le niveau de confiance mutuelle entre les $\mathrm{OC}$ et la direction que pouvait permettre leur proximité en CLSC. Dans le milieu des ICDC, tout en considérant que l'engagement du CSSS n'a pas fléchi quant au soutien des organismes communautaires, on estime que l'importance accrue des règles administratives rend l'accès plus difficile aux contributions de l'organisation communautaire.

Quant aux cadres, conscients de cette situation, ils estiment que l'expertise des OC doit se faire reconnaître dans les CSSS. Les OC ont à faire leur place dans l'établissement en s'inscrivant dans la nouvelle donne des rapports CSSS - milieu. Si les OC sont utiles pour savoir ce que veut le milieu, ils ne sont plus les seuls à disposer de cette information. Ce sont souvent les directions générales ou des cadres supérieurs qui siègent dans les instances locales de concertation. Les interlocuteurs des organismes communautaires dans les CSSS ne sont plus seulement les OC, même si ceux-ci demeurent, pour les dirigeants des ICDC, des complices et une source précieuse d'informations stratégiques. II n'y a pas du côté des directions de CSSS la même complicité et l'apport professionnel des OC n'est pas nécessairement pris en compte pour améliorer les rapports de l'établissement avec son milieu.

Dans ce contexte, les cadres identifient deux enjeux majeurs pour l'organisation communautaire: d'une part, les OC ont à faire la preuve dans leur CSSS que l'organisation communautaire doit être valorisée comme pratique professionnelle même si en nombre ils constituent un groupe relativement petit dans l'ensemble du personnel d'un CSSS; d'autre part, les cadres des CSSS, en particulier ceux qui ne 
sont pas issus des CLSC, ont besoin de comprendre ce que signifie travailler avec le milieu. Les attentes des cadres à l'égard des OC sont de l'ordre du partage de leur connaissance fine du territoire et du soutien à la mise en œuvre de la responsabilité populationnelle de l'établissement dans le cadre d'une redéfinition des concertations et des partenariats locaux. Cela passe par la transmission d'informations, la formation des intervenants à l'approche communautaire et le renforcement des liens des avec les milieux de la formation et de la recherche pour asseoir leur expertise sur un corpus de connaissances qui en valide le caractère scientifique. Les cadres locaux de référence et de pratique en organisation communautaire constituent, de ce point de vue, une occasion d'établir la crédibilité de l'organisation communautaire dans l'établissement (Bourque, 2009). Là où il n'y a pas d'espace de débat entre les OC pour développer de la cohésion sur les enjeux communautaires et sur les modèles de pratique, la reconnaissance de leurs interventions est compromise. La pratique de l'organisation communautaire est, par définition, une pratique frontière et les OC sont habitués à ce qu'elle suscite des questionnements, voire soit menacée. Ils devraient donc avoir le réflexe de faire leur place dans les nouveaux CSSS.

Les OC déplorent surtout que leur CSSS soit installé sur un territoire qui leur fait perdre le rapport de proximité qui caractérisait les CLSC, et que leurs tâches comportent moins de temps passé dans le milieu pour en prendre le pouls et identifier les attentes. Ils estiment nécessaire de se donner les moyens de garder le contact avec la population et certains craignent de devenir, selon leur expression, des « technocrates de programmes-cadres venant d'en haut ». Le fait que leur pratique soit soumise à davantage de contraintes administratives et à des délais plus longs pour établir une cohésion entre les vues de la direction et les plans d'intervention des OC leur laisse moins de temps de présence sur le terrain. Toutefois, la responsabilité populationnelle, la nécessité d'établir un portrait du territoire et l'obligation de développer des rapports de partenariat avec les autres organisations, particulièrement les groupes communautaires, constituent des occasions de mettre en évidence l'apport de l'organisation communautaire à la mission du CSSS. Les OC considèrent que, d'une manière certaine, les CSSS ont besoin de l'organisation communautaire et ce point de vue est partagé par leurs cadres.

\section{DISCUSSION : UN PROFIL RENOUVELÉ DE L'ORGANISATION COMMUNAUTAIRE EN CSSS}

Le profil de l'organisation communautaire tel qu'il se dégage de l'enquête comporte quatre caractéristiques. On attend des OC que leur expertise en processus d'action communautaire favorise la participation citoyenne; qu'ils soient des accompagnateurs de milieu; qu'ils assument un leadership dans le sens du bien commun et qu'ils soient des agents de liaison et de médiation entre les partenaires du milieu.

\section{Expertise en processus et participation citoyenne}

En développement des communautés, la participation est déterminante : intervenir par et avec la communauté distingue le développement des communautés des programmes pensés pour la communauté par des experts estimant savoir ce qui répond aux besoins. Les OC doivent donc se donner les moyens de garder le contact 
avec la population de façon à demeurer les gardiens et les soutiens de la participation des citoyennes et citoyens.

Les attentes du milieu à l'égard des OC portent à la fois sur leur savoir-faire dans la mise en œuvre des processus d'action collective et leur expertise quant au milieu. On apprécie leur souci d'amener les gens à faire un diagnostic continu, leur préoccupation que les gens aient du plaisir à le faire et ne cherchent pas passer trop vite à l'action, bref, qu'ils s'inspirent de l'approche d'action-réflexion-action de l'éducation populaire.

\section{Accompagnateurs de milieu}

Nous sommes passés d'une période de développement de ressources communautaires - grosso modo la période d'implantation des CLSC et les deux décennies qui ont suivi - à une période de développement de communautés. Alors que les OC, durant les années 1970 et 1980, avaient davantage investi dans la mobilisation de citoyens et le soutien à la création de nouvelles ressources, il y a maintenant moins de démarrages de groupes et ce sont plutôt les groupes en place ou les nouvelles ICDC qui développent des projets communautaires. La redéfinition de la profession doit prendre en compte la maturité de ces organismes et l'organisation communautaire doit être reconnue pour ce qu'elle est, une offre de services au milieu qui répond aux exigences de l'approche populationnelle des CSSS et du développement durable des communautés. On s'attend donc à ce que les OC soutiennent la participation aux démarches de concertation et de développement local des groupes dans lesquels les citoyens sont actifs. Les $\mathrm{OC}$ se voient comme des accompagnateurs qui croient au potentiel des personnes et des communautés, plutôt que comme des experts. Ils doivent savoir être en mode de proposition sans se substituer au milieu qui doit demeurer maître de ses choix; en somme, être, selon le mot d'un OC, « des accompagnateurs de milieu » plutôt que des accompagnateurs de groupes. Cette notion d'accompagnement de milieu ajoute aux pratiques des OC une dimension qui répond à la fois aux attentes des communautés en développement durable et à celles des CSSS dont la responsabilité populationnelle appelle une telle compétence.

\section{Leadership et bien commun}

En termes de leadership, les OC sont reconnus comme des intervenants centrés sur le milieu et capables de largeur de vue. Comme le dit un responsable d'ICDC : « De la façon dont ils sont positionnés, ils sont à même de voir globalement les besoins du milieu. » On s'attend à ce « qu'ils aient des positions sur les enjeux du quartier, annoncent leurs couleurs et contribuent au débat». On souhaite qu'ils aident à identifier les enjeux de lutte contre la pauvreté et les défis posés par les déterminants sociaux de la santé, mais aussi à dépasser la concertation sectorielle ou thématique pour intégrer les contributions de plusieurs réseaux. Ces processus complexes appellent un leadership de milieu que les dirigeants des ICDC reconnaissent aux OC. Cela rejoint les résultats de l'enquête de Comeau et al. (2008), selon laquelle le tiers des OC se sentent à l'aise sur ce terrain des coalitions et des réseaux et un OC sur cinq affirme intervenir dans le cadre de tables de concertation. On peut raisonnablement considérer que, depuis cette enquête réalisée en 2004 , la proportion d'OC soutenant des démarches de concertation a connu une croissance importante. Plusieurs ont souhaité que les OC exercent un leadership rassembleur qui renvoie aux 
compétences que le Programme national de santé publique considère comme des facteurs clés de l'action intersectorielle: «l'engagement envers la population et les diverses clientèles », la connaissance et « la reconnaissance de l'expertise des autres secteurs » et « une vision stratégique et l'aptitude à saisir les occasions » (Québec, 2008 , p. 67). On attend des OC qu'ils soient porteurs de perspectives larges et clairement établies, qui dépassent et englobent les initiatives sectorielles. Du fait de la durée de leur présence dans les communautés, on leur reconnaît une fonction de mémoire aussi bien de l'action communautaire des groupes que des concertations dans leur milieu. Cette perspective historique est essentielle pour l'analyse des enjeux.

\section{Liaison et médiation}

Les OC se perçoivent eux-mêmes comme des catalyseurs, des agents de liaison qui, comme le dit l'un deux, savent « voir comment chacun des partenaires va y trouver son compte » et rallier des acteurs de cultures organisationnelles différentes. Ce sont des intervenants habitués à vivre avec les exigences d'un métier qui se pratique aux frontières. Encore faut-il réunir les conditions pour que cela soit possible, aussi bien en termes de rapports de confiance entre la direction et les OC qu'en termes d'engagement du CSSS en développement des communautés. C'est certainement un enjeu actuel des processus de définition et d'encadrement de l'organisation communautaire dans les CSSS.

C'est un enjeu aussi pour les ICDC, qui disposent de nouvelles ressources financières pour intervenir sur les problématiques d'appartenance communautaire, de solidarité sociale et de revitalisation de milieux mal développés, mais qui dépendent encore de financements sur mesure donc relativement précaires. Pour demeurer des partenaires majeurs des institutions; capables de générer de nouvelles réponses aux besoins collectifs de ces milieux, de susciter de nouveaux leaderships et de créer de nouveaux outils de développement des communautés, elles comptent sur le soutien qui peut venir des CSSS, notamment des rapports de complicité avec les OC. Leur action repose sur des démarches de concertation dans lesquelles elles partagent avec les OC une compétence d'animation citoyenne et d'action communautaire.

\section{L'ENGAGEMENT DES CSSS EN DÉVELOPPEMENT DES COMMUNAUTÉS}

L'organisation communautaire en CSSS s'ajuste à une nouvelle génération d'action communautaire qui s'affirme depuis que l'État québécois a reconnu la contribution de l'action communautaire à l'action citoyenne. Mais elle se cherche aussi : si les rapports avec les organismes communautaires demeurent solides, le développement des communautés n'est pas une évidence dans les CSSS. Le discours de la santé publique (Québec, 2008) est à cet égard en avance sur les pratiques du réseau.

Une indication que le développement des communautés n'est pas clairement établi dans les CSSS réside dans la décision de certaines directions de ne pas favoriser la participation des OC à des formations spécifiques. Dans certains CSSS, en effet, les OC se voient interdire de participer à des rencontres à l'extérieur du territoire, notamment aux rencontres régionales et nationales du Regroupement québécois des intervenantes et intervenants en action communautaire en CSSS (RQIIAC). 
L'argument selon lequel cela ne se fait pas dans les autres professions, invoqué par certaines directions, manifeste une méconnaissance des exigences de l'action communautaire. Le soutien aux démarches de développement des communautés ne se limite pas à la mise en œuvre de techniques, mais exige une analyse des enjeux sociaux et politiques, qui ne peut se développer que dans des interactions à de multiples niveaux. La promotion de politiques publiques est constitutive du développement des communautés (Cadre de référence en santé publique pour le développement des communautés, 2002) et passe nécessairement par des débats et des interventions à l'échelle des régions où les conférences régionales des élus (CRÉ) et les agences de la santé et des services sociaux assurent l'application des politiques québécoises, voire à l'échelle des travaux de l'Assemblée nationale. Ce fut le cas, par exemple, pour la Loi visant à lutter contre la pauvreté et l'exclusion sociale (LRQ, ch. L-7) dont l'adoption est le résultat d'un long processus d'organisation communautaire dans le cadre du Collectif pour un Québec sans pauvreté auquel le RQIIAC a contribué, et que plusieurs CLSC ont appuyé.

\section{EN GUISE DE CONCLUSION}

La quasi-totalité des entrevues réalisées affirment la pertinence de la contribution de l'organisation communautaire aux ICDC. Dans leurs interventions les OC sont capables de distance critique, ils amènent les démarches à se centrer sur le milieu et renouvellent le soutien à l'action communautaire. On attend d'eux un apport spécifique pour l'analyse des déterminants sociaux de la santé, notamment parce qu'ils ont une bonne connaissance du milieu et qu'ils ont accès à l'expertise en santé publique. On compte sur eux aussi pour la formation de nouveaux leaders et pour le soutien à la participation citoyenne.

En contrepartie, le statut des OC dans les CSSS fait l'objet de préoccupations dans la majorité des entrevues. Plusieurs de nos répondants considèrent que la reconfiguration du réseau comporte des menaces pour l'organisation communautaire et identifient chez les OC des comportements de résistance qui ne sont pas de nature à faciliter les choses. La grande majorité des entrevues ont fait ressortir le changement institutionnel que constituent la création des CSSS et la nécessité qui en découle de redéfinir l'organisation communautaire : établissement de nouveaux rapports avec la direction, modification de l'encadrement professionnel et du fonctionnement en équipe, et davantage d'orientations venant d'«en haut». Certains directeurs de CSSS estiment que les OC ne sont plus essentiels puisqu'eux-mêmes assument les relations de partenariat. Cette tendance a été exprimée de façon minoritaire dans les entrevues, tout comme elle se manifeste de façon minoritaire dans les CSSS qui ont choisi de transformer les postes d'OC en postes d'agent de programmation et de développement. Généralement toutefois, on apprécie que les OC demeurent les complices du communautaire et on espère le développement de mécanismes permettant que cela soit un plus pour les CSSS.

Enfin, avec des agents de la santé publique qui font la promotion du développement des communautés, il y a un terrain d'alliances à développer. Comme le souligne un cadre : 
Il y a des gens à l'interne de la Santé publique qui défendent les mêmes positions que l'organisation communautaire, qui pensent qu'il faut partir de la communauté malgré le beau programme qu'ils ont en main, et qu'on doit l'adapter. Ces gens-là ont besoin de soutien et certainement pas qu'on balaie de la main toute la santé publique.

Les OC auraient donc avantage à repenser leurs rapports aux instances de la santé publique et pourraient appliquer avec les agents des directions régionales de santé publique la stratégie qui leur a permis, selon Comeau et al. (2008), non seulement de maintenir, mais de renforcer leur présence dans les CLSC : mettre à profit leur savoirfaire pour susciter et soutenir des alliances entre des partenaires dont les perspectives sont parfois différentes, mais dont on souhaite faire des alliés pour le profit des communautés.

Les processus en cours de mise à jour ou d'élaboration de cadres de référence locaux en organisation communautaire peuvent contribuer à la reconnaissance des pratiques communautaires dans les CSSS où la culture des CLSC demeure relativement marginale. L'existence des ICDC manifeste un saut qualitatif dans la contribution des acteurs locaux et en particulier du milieu communautaire au développement des communautés. Les apports attendus des OC dans ces démarches exigent que les pratiques s'ajustent mieux aux défis actuels des milieux, défis de cohésion sociale, mais aussi défi de redéfinition du développement en fonction des enjeux du $21^{\mathrm{e}}$ siècle, notamment ceux qui ont été établis par le programme Action 21 de l'Organisation des Nations unies que l'on peut consulter en ligne [http://www.un.org/french/events/rio92/agenda21/action0.htm], et par le rapport de la Commission de l'OMS sur les déterminants sociaux de la santé (CSHD, 2008) qui propose comme priorité politique de réduire en une génération le fossé des inégalités sociales de santé.

\section{BIBLIOGRAPHIE}

BOURQUE, Denis (2009). «Cadres de référence en organisation communautaire », Interaction communautaire, $\mathrm{n}^{\circ} 80$, hiver, p. 17-18.

BRUNET, Lyse et Pierre DUROCHER (2006). « Un quartier qui veut s'en sortir : I'histoire de la mobilisation dans Saint-Michel », dans Michel VENNE et Miriam FAHMY (sous la direction de), L'Annuaire du Québec. Le Québec en panne ou en marche?, Fides, p. 312-317.

CADRE DE RÉFÉRENCE EN SANTÉ PUBLIQUE POUR LE DÉVELOPPEMENT DES COMMUNAUTÉS, (2002). La santé des communautés : perspectives pour la contribution de la santé publique au développement social et au développement des communautés, Institut national de santé publique du Québec, 50 p.

Comeau, Yvan, Martine Duperré, Yves Hurtubise, Clément Mercier et Daniel TURCOTTE (2006). « Les transformations de l'organisation communautaire en CSLC (1988-2004) », Inter-action communautaire, $n^{0} 73$, automne, p. 18-21. 
Comeau, Yvan, Martine Duperré, Yves Hurtubise, Clément Mercier et Daniel TURCOTTE (2008). L'organisation communautaire en mutation. Étude sur la structuration d'une profession du social, Presses de l'Université Laval, 194 p.

CSDH (2008). Closing the gap in a generation: health equity through action on the social determinants of health. Final report of the Commission on social determinants of health, Genève, Organisation mondiale de la santé, 248 p.

DAUNAIS, Jean-Paul (1986). « L'entretien non directif », dans Benoît GAUTHIER (sous la direction de) (1987), Recherche sociale, de la problématique à la collecte des données, Presses de l'Université du Québec, p. 247-275.

ElLyson, A. et D. BourQue (2009). «Projets cliniques territoriaux des CSSS et participation des acteurs de la société civile », Organisations et territoires, vol. 18, $\mathrm{n}^{\mathrm{o}}$ 1, p. 77-86.

FAVREAU, Louis (2008). Entreprises collectives. Les enjeux sociopolitiques et territoriaux de la coopération et de l'économie sociale, Québec, Presses de l'Université du Québec, 332 p.

FAVREAU, Louis et Yves HURTUBise (1993). CLSC et communautés locales: la contribution de l'organisation communautaire, Québec, Presses de l'Université du Québec, 211 p.

FoISY, Dominic (2004). L'organisation communautaire en CLSC: au carrefour de légitimités renouvelées, Mémoire présenté comme exigence partielle de la maîtrise en travail social, Université du Québec en Outaouais, juin, 143 p.

FoIsY, Dominic, Denis BouRQue et René LACHAPELLE (2009). La coordination professionnelle des équipes en organisation communautaire en CSSS : description, perspectives et enjeux, CRCOC, $\mathrm{n}^{\circ}$ 0901, janvier, $56 \mathrm{p}$.

FOURNIER, J. (2009). « Le mouvement communautaire s'autocensure-t-il à cause du financement gouvernemental? », Le Devoir, mardi 11 août 2009.

JetTÉ, Christian (2006). Pistes de réflexion pour le développement de l'action communautaire au $X X I^{e}$ siècle, Cahiers du LAREPPS $n^{0} 06-13$, École de travail social, Université du Québec à Montréal, décembre, $45 \mathrm{p}$.

JETTÉ, Christian (2008). Les organismes communautaires et la transformation de l'Étatprovidence. Trois décennies de coconstruction des politiques publiques dans le domaine de la santé et des services sociaux, Québec, Presses de l'Université du Québec, $422 \mathrm{p}$.

LACHAPELLE, René (sous la direction de) (2003). L'organisation communautaire en CLSC. Cadre de référence et pratiques, Les Presses de l'Université Laval, 293 p.

QUÉBEC (1988). Rapport de la Commission d'enquête sur les services de santé et les services sociaux, Les Publications du Québec, 803 p.

QUÉBEC (2000). Les solutions émergentes. Rapport et recommandations de la Commission d'étude sur les services de santé et les services sociaux, 408 p. 
QUÉBEC (2001). L'action communautaire, une contribution essentielle à l'exercice de la citoyenneté et au développement social du Québec, Politique gouvernementale, septembre, $60 \mathrm{p}$.

QUÉBEC (2004b). Les services généraux offerts par les centres de santé et de services sociaux, septembre, $179 \mathrm{p}$.

QUÉBEC (2008). Programme national de santé publique 2003-2012. Mise à jour 2008, Québec, Santé et Services sociaux, 103 p.

RoBItAILle, M. (2007). Les métiers du développement local et régional au Québec: l'émergence de nouvelles compétences, Cahier du CRDT, Série Recherches, $\mathrm{n}^{\circ} 9$, $31 \mathrm{p}$.

SHRAGGE, Eric (2006). Action communautaire : dérives et possibles, Écosociété, 242 p.

SHRAGGE, Eric (2007). « La pratique communautaire et la lutte pour la transformation sociale », Nouvelles pratiques sociales, vol. 19, $\mathrm{n}^{\circ} 2$, p. 182-192.

THÉRIAULT, Sylvain (sous la direction de) (2004). « Dossier : Les urbanistes ont-ils peur de la revitalisation urbaine intégrée? », Urbanité, novembre, p. 15-33. 